\title{
Inadequação calórica e proteica e fatores associados em pacientes graves
}

\author{
Inadequate energy and protein and associate \\ factors in critically ill patients
}

Mariana de Melo Alves SANTANA ${ }^{1}$

Liana Lima VIEIRA²

Daniela de Araújo Medeiros DIAS 3

Cinthya Costa BRAGA ${ }^{1}$

Raphaela Moiana da COSTA $^{1}$

\section{R E S U M O}

\section{Objetivo}

Investigar a adequação calórico-proteica de pacientes em terapia nutricional enteral exclusiva internados nas Unidades de Terapia Intensiva de um hospital universitário.

\section{Métodos}

Estudo longitudinal realizado entre abril e novembro de 2014 nas Unidades de Terapia Intensiva de um hospital universitário. Foram avaliados no período de 14 dias: percentual de adequação calórico-proteica, calculada pelas médias dos valores prescritos e administrados; condições clínicas (unidade e diagnóstico de internação, escore prognóstico Acute Physiology and Chronic Health Evaluation II, estado nutricional, tempo de internação); complicações gastrointestinais; e motivo de interrupção da dieta. Valores inferiores a 80\% de adequação calórica e proteica foram considerados inadequados. Realizou-se análise multivariada por Regressão de Poisson com nível de significância de 5\%.

\section{Resultados}

O estudo contou com uma amostra de 38 pacientes, sendo 52,63\% desnutridos. As médias de adequação calórica e proteica foram de 76,47\% e 69,11\%, respectivamente. A prevalência de inadequação calórica foi de $55,26 \%$ e de proteica, 68,42\%. O jejum para procedimentos foi a causa mais frequente de interrupção da dieta. O volume residual gástrico e a diarreia foram as complicações gastrointestinais mais comuns. A inadequação calórica associou-se ao tempo de permanência $\leq 14$ dias e ao volume residual elevado. A inadequação proteica associou-se ao tempo de permanência $\leq 14$ dias, ao volume residual gástrico elevado e à saída ou obstrução da sonda.

\footnotetext{
1 Universidade Federal de Goiás, Hospital das Clínicas, Programa de Residência Multiprofissional em Saúde, Terapia Intensiva. $1^{a}$ Avenida, s/n., Setor Leste Universitário, 74605-020, Goiânia, GO, Brasil. Correspondência para/Correspondente to: MMA SANTANA.E-mail:<marinutri@live.com.br>.

${ }^{2}$ Universidade Federal de Goiás, Hospital das Clínicas, Departamento de Nutrição. Goiânia, GO, Brasil.

3 Centro Universitário Unieuro, Escola da Saúde, Curso de Nutrição. Brasília, DF, Brasil.
} 


\section{Conclusão}

A prevalência de inadequação calórica e proteica ocorreu em mais da metade dos pacientes avaliados. Estratégias como a elaboração de protocolos por equipe multiprofissional devem ser implantadas para minimizar interrupções da dieta administrada, estabelecer medidas de controle para complicações gastrointestinais e, assim, garantir um aporte nutricional adequado durante o período de internação.

Palavras-chave: Cuidados críticos. Ingestão de energia. Necessidade energética. Nutrição enteral. Terapia nutricional.

\section{A B S T R A C T}

\section{Objective}

To investigate the energy and protein adequacies of intensive care unit of a university hospital patients in exclusive enteral nutrition.

\section{Methods}

This longitudinal study was conducted between April and November 2014 in the adult intensive care unit of a university hospital. The following items were assessed during 14 days: percentage of protein energy adequacy, based on the mean prescribed and administered values; clinical conditions (unit and diagnosis on admission, Acute Physiology and Chronic Health Evaluation II, nutritional status, and length of stay); gastrointestinal complications; and reason for diet interruption. Values below $80 \%$ of the caloric and protein adequacies were considered inadequate. Multivariate analysis was performed by Poisson Regression at a significance level of $5 \%$.

\section{Results}

The sample consisted of 38 patients, and $52.63 \%$ were undernourished. The mean caloric and protein adequacies were $76.47 \%$ and $69.11 \%$, respectively. The prevalences of caloric and protein inadequacies were $55.26 \%$ and $68.42 \%$, respectively. Fasting for procedures was the most frequent cause for diet interruption. Residual gastric volume and diarrhea were the most common gastrointestinal complications. Caloric inadequacy was associated with length of stay $\leq 14$ days and high residual gastric volume. Protein inadequacy was associated with length of stay $\leq 14$ days, high residual gastric volume, and catheter displacement or obstruction.

\section{Conclusion}

More than half of the patients had caloric and protein inadequacies. Strategies, such as the development of protocols for multidisciplinary teams, should be created to minimize disruption of the administered diet, establish measures to control gastrointestinal complications, and thus ensure adequate nutritional intake during intensive care unit stay.

Keywords: Critical care. Energy intake. Energy requirement. Enteral nutrition. Nutrition therapy.

\section{N T R O D U Ç Ã O}

A desnutrição é um dos maiores problemas em pacientes hospitalizados, principalmente em uma Unidade de Terapia Intensiva (UTI) ${ }^{1}$. Entre os pacientes críticos, existe uma prevalência de desnutrição superior a 35\% já na admissão em UTI. Isso também ocorre dentro do ambiente hospitalar, porém, na maioria das vezes, com maior intensidade devido à coexistência de fatores de risco ${ }^{2}$, variando entre 43 e $88 \%{ }^{3}$. Nesse contexto, a desnutrição tem sido relacionada ao aumento do risco de morbimortalidade e do tempo de internação ${ }^{4}$. O paciente crítico é tipicamente associado a um estado de estresse catabólico e a uma resposta inflamatória sistêmica. Essa resposta está relacionada a complicações que levam ao aumento da morbidade infecciosa, da disfunção múltipla de órgãos, da hospitalização prolongada e da taxa de mortalidade ${ }^{2-5}$.

A terapia nutricional em pacientes críticos é fundamental na recuperação do seu estado clínico, pois contribui para preservação da massa magra corporal e evita complicações metabólicas, 
colaborando na diminuição do estresse fisiológico e modulando a resposta imunológica. Dessa maneira, a resposta metabólica ao estresse diminui, evitando a lesão celular oxidativa ${ }^{1-6} \mathrm{O}$ início precoce da Terapia Nutricional Enteral (TNE), dentro de 24 a 48 horas após a admissão, em pacientes hemodinamicamente estáveis tem sido recomendado, sendo que o alcance das necessidades deverá acontecer em 48-72 horas $^{7}$. Evidências sugerem que a TNE auxilia na manutenção da integridade da mucosa intestinal, na diminuição da translocação bacteriana, na atenuação da resposta inflamatória de fase aguda mediada por citocinas, na diminuição dos riscos de infecções e na possível redução do risco de desenvolvimento de falência orgânica múltipla ${ }^{5-7}$.

No entanto, a adequação do suporte nutricional enteral pode ser prejudicada. Isso porque podem ocorrer diversas condições as quais interferem na oferta nutricional planejada, causando sua suspensão temporária e/ou permanente e favorecendo o declínio do estado nutricional ${ }^{8}$. Dentre essas condições estão a disfunção gastrointestinal (vômito, diarreia, distensão abdominal, resíduo gástrico aumentado), o jejum para exames e procedimentos, a remoção de sonda e a instabilidade clínica do paciente ${ }^{9-11}$. Esses fatores contribuem para que sujeitos em terapia intensiva estejam em risco de receber menor volume e aporte energético-proteico do que o prescrito ${ }^{12}$.

$\mathrm{O}$ débito energético acumulado é descrito como um forte preditor de desfechos clínicos, expondo os pacientes a deficit energéticos que, provavelmente, não serão compensados durante a internação nas Unidades de Terapia Intensiva ${ }^{13,14}$ A resposta metabólica ao estresse associada à imobilidade prolongada e à oferta nutricional inadequada potencializam o catabolismo e predispõe o deficit nutricional ${ }^{14,15}$. Dessa forma, a adequação da oferta energético-proteica ao paciente crítico representa um importante desafio.

A monitorização diária da oferta nutricional real é um instrumento para identificação das causas da administração de dieta enteral abaixo do planejado ${ }^{16}$. Assim, o objetivo do pre- sente estudo foi investigar a adequação calórico-proteica de pacientes em terapia nutricional enteral exclusiva internados nas Unidades de Terapia Intensiva de um hospital universitário.

\section{M É T O D O S}

Trata-se de um estudo longitudinal realizado no período de abril a novembro de 2014 nas UTI clínica e cirúrgica do Hospital das Clínicas da Universidade Federal de Goiás, Goiânia (GO). O projeto de pesquisa foi aprovado pelo Comitê de Ética em Pesquisa da instituição (Processo $n^{\circ} 265.518$ ) e os pacientes foram incluídos na pesquisa somente depois de assinado o Termo de Consentimento Livre e Esclarecido.

Participaram do estudo pacientes admitidos nas UTI, de ambos os sexos, apresentando idade igual ou superior a 18 anos e que receberam TNE exclusiva por, pelo menos, 72 horas. Os critérios de exclusão foram: indivíduos que estavam se alimentando pelas vias oral, parenteral ou enteral associada a outras vias de alimentação; pacientes em cuidados paliativos; e gestantes.

A coleta de dados foi iniciada no primeiro dia de introdução da nutrição enteral e o acompanhamento dos pacientes foi realizado até o momento de descontinuação da terapia nutricional, óbito, alta da unidade ou quando completados 14 dias com a dieta. Em até 48 horas, foram coletados os dados demográficos (sexo, idade), clínicos (diagnóstico de internação na UTI, escore prognóstico Acute Physiology and Chronic Health Evaluation II (APACHEII)) e classificação da Avaliação Subjetiva Global (ASG). A criticidade do paciente foi medida pelo APACHEII nas seguintes categorias $<15,15-23$, $>23$ pontos e o tempo de UTI foi avaliado entre os que permaneceram $\leq 14$ dias ou $>14$ dias $^{17}$.

A verificação do volume diário de dieta infundida, variáveis de interrupção do fornecimento da dieta enteral e complicações gastrintestinais foram acompanhados a partir de um formulário padronizado da enfermagem. As complicações gastrointestinais foram definidas como: 
vômito (ocorrência de um ou mais episódios em 12 horas) $^{8}$, diarreia (3 ou mais evacuações líquidas em 24 horas $^{8}$, volume residual gástrico ( $\geq 400 \mathrm{~mL}$ em 24 horas) $^{18}$ e presença de distensão abdominal. Os motivos de interrupção da terapia nutricional enteral analisados foram: jejum para procedimentos, exames ou cirurgias, presença de sintomas gastrointestinais e saída ou obstrução da sonda.

Para diagnóstico nutricional dos pacientes, empregou-se a avaliação subjetiva global, aplicada pelo pesquisador, sendo que o modelo utilizado foi o proposto por Detsky et al. ${ }^{19}$. Dessa for$\mathrm{ma}$, os pacientes puderam ser divididos em desnutridos (ASG-B e ASG-C) e nutridos (ASG-A).

As necessidades energéticas e proteicas foram estimadas individualmente, de acordo com a condição clínica do paciente a partir das recomendações da American Society for Parenteral and Enteral Nutrition (ASPEN) ${ }^{7}$. Utilizou-se, para o cálculo da adequação energética e proteica, 25 $\mathrm{kcal} / \mathrm{kg}$ peso corporal atual e 1,2 g proteína/kg peso corporal atual. Dessa maneira, para índices de massa corporal maiores que $30 \mathrm{~kg} / \mathrm{m}^{2}$, utilizou-se $11 \mathrm{kcal} / \mathrm{kg}$ peso corporal atual e $2 \mathrm{~g}$ proteína/ $\mathrm{kg}$ peso ideal. O peso corporal atual utilizado para o cálculo das necessidades foi estimado conforme equações propostas por Chumlea et al. ${ }^{20}$ quando da ausência do peso aferido ou referido.

Para cada paciente avaliado, foram verificadas, ao longo de 14 dias a partir do quarto dia de dieta enteral, a energia e a proteína efetivamente ofertadas pela TNE em relação às reais necessidades estimadas. Esse período foi assim determinado por ser o tempo necessário para a progressão do volume e alcance das necessidades nutricionais. A adequação calórico-proteica foi avaliada com base nas necessidades energética e proteica diárias comparadas com a média de valores energéticos e proteicos administrados diariamente, já que calorias e proteínas prescritas condiziam com as necessidades nutricionais. A porcentagem de adequação foi calculada pela relação percentual entre as médias dos valores prescritos e dos administrados a partir do quarto dia de implementação da terapia nutricional enteral. Considerou-se adequado um percentual de adequação calórica e adequação proteica $\geq 80 \%{ }^{21,22}$. Valores inferiores a $80 \%$ de adequação foram considerados inadequados.

A dieta enteral foi administrada continuamente em bomba de infusão durante, aproximadamente, 20 horas do dia. As quatro horas restantes foram reservadas para a realização de outros procedimentos médicos, fisioterapia, exames e administração de medicamentos.

A análise estatística dos dados foi processada no programa Stata versão 12.0 (Stata Corporation, College Station, Texas, Estados Unidos). Foi calculada a prevalência de ambos os desfechos (inadequação calórica e inadequação proteica) para cada variável analisada. Inicialmente, realizou-se a regressão de Poisson simples (estimativa robusta da variância) com o cálculo das Razões de Prevalência (RP) e respectivos Intervalos de Confiança de 95\% (IC95\%), considerando nível de significância de 5\%.

As variáveis que na análise bivariada inicial (para ambos os desfechos) apresentaram valor $p<0,20$ foram incluídas na análise multivariada por regressão de Poisson. Aquelas que apresentaram valor de $p$ acima de 0,05 foram retiradas do modelo multivariado. Já aquelas com valor de $p<0,05$ foram consideradas independentemente associadas a inadequação calórica e proteica.

\section{RES U L T A D OS}

Foram avaliados 38 pacientes com TNE exclusiva, com idade média de $61,71 \pm 14,72$ anos. O escore APACHE II médio foi de 17,63 $\pm 6,86$. O tempo médio de internação na UTI foi de $16,47 \pm 10,13$ (mediana=12) dias, sendo que $63,16 \%$ dos pacientes permaneceram por tempo $\leq 14$ dias. A TNE foi utilizada por um período médio de $13,87 \pm 11,54$ dias.

O gasto energético total médio foi de $1438,24 \pm 342,22 \mathrm{kcal} / \mathrm{dia}$ e a necessidade proteica média foi de 77,69 21,22 g/dia. A média 
de energia ofertada foi $1053,06 \pm 366,37 \mathrm{Kcal} / \mathrm{dia}$ e de proteína, 52,34 $\pm 20,65 \mathrm{~g} / \mathrm{dia}$, o que representa, respectivamente, uma adequação de $76,47 \pm 29,57 \%$ e $69,11 \pm 25,37 \%$.

Os principais diagnósticos de internação foram: pós-operatório $(n=11)$, respiratório $(n=10)$, sepse grave/choque séptico $(n=8)$, neurológico $(n=4)$, gastroenterológico $(n=3)$ e neoplasia $(n=2)$. A descrição das demais variáveis estudadas encontra-se em distribuição na amostra ( $n$ e \%) nas Tabelas 1 e 2.

As prevalências de inadequação calórica e proteica na amostra foram de 55,26\% e 68,42\%, respectivamente. A média do tempo para atingir a meta de adequação calórica foi de 2,62 11,47 dias e para adequação proteica, 3,75 $\pm 2,25$ dias.

Quanto às variáveis clínicas e nutricionais, observou-se que as maiores prevalências de inadequação calórica foram para o sexo masculino, idade entre 60 e 69 anos, na categoria de APACHE II >23 pontos, em unidade de internação clínica e para aqueles classificados como desnutridos, porém essas variáveis não estão associadas entre si na análise bivariada. No entanto, a inadequação proteica foi 1,94 vezes maior (IC95\%:1,02-3,68) em pacientes com tempo de permanência $\leq$ a 14 dias, quando comparados aos pacientes com tempo de permanência $>14$ dias. Demais variáveis clínicas e nutricionais não estiveram associadas à inadequação proteica (Tabela 1).

Os sintomas gastrointestinais foram observados em $50 \%$ dos pacientes e, dentre eles, destacaram-se o Volume Residual Gástrico (VRG) e a diarreia, com frequências semelhantes (28,95\%); o vômito $(18,42 \%)$; e a distensão abdominal $(15,79 \%)$. Quanto às intercorrências relacionadas à terapia nutricional, $86,84 \%$ dos pacientes apresentaram alguma intercorrência que levou à interrupção da dieta. Dentre os motivos para essa interrupção, o jejum para procedimentos apresentou a maior frequência na amostra (60,53\%). $\mathrm{Na}$ análise bivariada, a inadequação calórica

Tabela 1. Distribuição na amostra, Razão de Prevalência (RP) e Intervalo de Confiança (IC) da inadequação calórica e proteica segundo variáveis clínicas e nutricionais. Hospital das Clínicas da Universidade Federal de Goiás, Goiânia (GO), 2015 (n=38).

\begin{tabular}{|c|c|c|c|c|c|c|c|c|c|c|}
\hline \multirow{3}{*}{ Variáveis } & \multirow{2}{*}{\multicolumn{2}{|c|}{$\begin{array}{c}\text { Distribuição na } \\
\text { amostra }\end{array}$}} & \multicolumn{4}{|c|}{ Inadequação calórica } & \multicolumn{4}{|c|}{ Inadequação proteica } \\
\hline & & & \multicolumn{2}{|c|}{ Prevalência } & \multirow{2}{*}{ RP(IC95\%) } & \multirow{2}{*}{ Valor $p$} & \multicolumn{2}{|c|}{ Prevalência } & \multirow{2}{*}{ RP(IC95\%) } & \multirow{2}{*}{ Valor $p$} \\
\hline & $n$ & $(\%)$ & $\mathrm{n}$ & $(\%)$ & & & $\mathrm{n}$ & $(\%)$ & & \\
\hline Sexo & & & & & & 0,148 & & & & 0,666 \\
\hline Feminino & 17 & 44,74 & 7 & 41,18 & 1,00 & & 11 & 64,71 & 1,00 & \\
\hline Masculino & 21 & 55,26 & 14 & 66,67 & $1,62(0,84-3,11)$ & & 15 & 71,43 & $1,10(0,70-1,72)$ & \\
\hline Idade & & & & & & 0,344 & & & & 0,262 \\
\hline $18-59$ & 16 & 42,10 & 8 & 50,00 & 1,00 & & 13 & 81,25 & $1,62(0,91-2,91)$ & \\
\hline $60-69$ & 8 & 21,05 & 6 & 75,00 & $1(0,48-2,06)$ & & 6 & 75,00 & $1,5(0,76-2,92)$ & \\
\hline$\geq 70$ & 14 & 36,85 & 7 & 50,00 & $1,5(0,77-2,92)$ & & 7 & 50,00 & 1,00 & \\
\hline APACHE /I & & & & & & 0,128 & & & & 0,976 \\
\hline$<15$ & 12 & 31,58 & 5 & 41,67 & 1,00 & & 8 & 66,67 & 1,00 & \\
\hline $15-23$ & 20 & 52,63 & 11 & 55,00 & $1,32(0,60-2,90)$ & & 14 & 70,00 & $1,05(0,64-1,73)$ & \\
\hline$>23$ & 6 & 15,79 & 5 & 83,33 & $2(0,92-4,31)$ & & 4 & 66,67 & $1,00(0,35-2,01)$ & \\
\hline Unidade de internação & & & & & & 0,693 & & & & 0,338 \\
\hline Cirúrgica & 21 & 55,26 & 11 & 52,38 & 1,00 & & 13 & 61,90 & 1,00 & \\
\hline Clínica & 17 & 44,74 & 10 & 58,82 & $1,12(0,63-2,00)$ & & 13 & 76,47 & $1,23(0,80-1,90)$ & \\
\hline Estado nutricional & & & & & & 0,227 & & & & 0,828 \\
\hline Nutridos & 18 & 47,37 & 8 & 44,44 & 1,00 & & 12 & 66,67 & 1,00 & \\
\hline Desnutridos & 20 & 52,63 & 13 & 65,00 & $1,46(0,79-2,71)$ & & 14 & 70,00 & $1,05(0,67-1,63)$ & \\
\hline Tempo de permanência UTI & & & & & & 0,111 & & & & 0,041 \\
\hline$\leq 14$ dias & 24 & 63,16 & 16 & 66,67 & $1,87(0,87-4,02)$ & & 20 & 83,33 & $1,94(1,02-3,68)$ & \\
\hline$>14$ dias & 14 & 36,84 & 5 & 35,71 & 1,00 & & 6 & 42,86 & 1,00 & \\
\hline
\end{tabular}

Nota: APACHEIl: Acute Physiology and Chronic Health Evaluation II; UTI: Unidade de Terapia Intensiva. 
Tabela 2. Distribuição na amostra, Razão de Prevalência (RP) e Intervalo de Confiança (IC) da inadequação calórica e proteica segundo variáveis de interrupção da dieta e complicações gastrointestinais. Hospital das Clínicas da Universidade Federal de Goiás, Goiânia (GO), 2015 ( $n=38)$.

\begin{tabular}{|c|c|c|c|c|c|c|c|c|c|c|}
\hline \multirow{3}{*}{ Variáveis } & \multirow{2}{*}{\multicolumn{2}{|c|}{$\begin{array}{c}\text { Distribuição na } \\
\text { amostra }\end{array}$}} & \multicolumn{4}{|c|}{ Inadequação calórica } & \multicolumn{4}{|c|}{ Inadequação proteica } \\
\hline & & & \multicolumn{2}{|c|}{ Prevalência } & \multirow{2}{*}{ RP(IC95\%) } & \multirow{2}{*}{ Valor $p$} & \multicolumn{2}{|c|}{ Prevalência } & \multirow{2}{*}{ RP(IC95\%) } & \multirow{2}{*}{ Valor $p$} \\
\hline & $\mathrm{n}$ & $(\%)$ & $n$ & $(\%)$ & & & $n$ & $(\%)$ & & \\
\hline Vômito & & & & & & 0,019 & & & & 0,169 \\
\hline $\operatorname{Sim}$ & 7 & 18,42 & 6 & 85,71 & $1,77(1,10-2,86)$ & & 6 & 85,71 & $1,33(0,89-1,99)$ & \\
\hline Não & 31 & 81,58 & 15 & 48,39 & 1,00 & & 20 & 64,52 & 1,00 & \\
\hline Diarreia & & & & & & 0,491 & & & & 0,708 \\
\hline Sim & 11 & 28,95 & 7 & 63,64 & $1,22(0,68-2,20)$ & & 8 & 72,73 & $1,09(0,69-1,72)$ & \\
\hline Não & 27 & 71,05 & 14 & 51,85 & 1,00 & & 18 & 66,67 & 1,00 & \\
\hline Distensão abdominal & & & & & & 0,790 & & & & 0,922 \\
\hline $\operatorname{Sim}$ & 6 & 15,79 & 3 & 50,00 & 1,00 & & 22 & 68,75 & $1,03(0,55-1,91)$ & \\
\hline Não & 32 & 84,21 & 18 & 56,25 & $1,12(0,47-2,68)$ & & 4 & 66,67 & 1,00 & \\
\hline Volume Residual Gástrico & & & & & & 0,002 & & & & 0,207 \\
\hline $\operatorname{Sim}$ & 11 & 28,95 & 10 & 90,91 & $2,23(1,35-3,67)$ & & 9 & 81,82 & $1,30(0,86-1,95)$ & \\
\hline Não & 27 & 71,05 & 11 & 40,74 & 1,00 & & 17 & 62,96 & 1,00 & \\
\hline Jejum para procedimentos & & & & & & 0,850 & & & & 0,854 \\
\hline $\operatorname{Sim}$ & 23 & 60,53 & 13 & 56,52 & $1,05(0,58-1,93)$ & & 16 & 69,57 & $1,04(0,66-1,64)$ & \\
\hline Não & 15 & 39,47 & 8 & 53,33 & 1,00 & & 10 & 66,67 & 1,00 & \\
\hline Exame ou cirurgia & & & & & & 0,256 & & & & 0,522 \\
\hline Sim & 16 & 42,11 & 7 & 43,75 & 1,00 & & 10 & 62,50 & 1,00 & \\
\hline Não & 22 & 57,89 & 14 & 63,64 & $1,45(0,76-2,78)$ & & 16 & 72,73 & $1,16(0,73-1,85)$ & \\
\hline Sintomas gastrointestinais & & & & & & 0,038 & & & & 0,494 \\
\hline $\operatorname{Sim}$ & 19 & 50,00 & 14 & 73,68 & $2,00(1,03-3,85)$ & & 14 & 73,68 & $1,16(0,75-1,81)$ & \\
\hline Não & 19 & 50,00 & 7 & 36,84 & 1,00 & & 12 & 63,16 & 1,00 & \\
\hline Saída/obstrução sonda & & & & & & 0,501 & & & & 0,001 \\
\hline $\operatorname{Sim}$ & 6 & 15,79 & 4 & 66,67 & $1,25(0,65-2,43)$ & & 6 & 100 & $1,60(1,21-2,10)$ & \\
\hline Não & 32 & 84,21 & 17 & 53,13 & 1,00 & & 20 & 62,50 & 1,00 & \\
\hline
\end{tabular}

Tabela 3. Modelo final de análise multivariada da inadequação calórica e proteica. Hospital das Clínicas da Universidade Federal de Goiás, Goiânia (GO), 2015.

\begin{tabular}{|c|c|c|c|}
\hline Variáveis & Razão de Prevalência & Intervalo de Confiança 95\% & Valor $p$ \\
\hline \multicolumn{4}{|c|}{ Inadequação calórica } \\
\hline \multicolumn{4}{|c|}{ Tempo de permanência UTI } \\
\hline$\leq 14$ dias & 2,07 & $1,11-3,87$ & 0,022 \\
\hline$>14$ dias & 1,00 & & \\
\hline \multicolumn{4}{|c|}{ Volume residual gástrico } \\
\hline Sim & 2,41 & $1,51-3,86$ & $<0,001$ \\
\hline Não & 1,00 & & \\
\hline \multicolumn{4}{|c|}{ Inadequação proteica } \\
\hline \multicolumn{4}{|c|}{ Tempo de permanência UTI } \\
\hline$\leq 14$ dias & 2,05 & $1,14-3,67$ & 0,016 \\
\hline$>14$ dias & 1,00 & & \\
\hline \multicolumn{4}{|c|}{ Volume residual gástrico } \\
\hline Sim & 1,54 & $1,05-2,26$ & 0,025 \\
\hline Não & 1,00 & & \\
\hline \multicolumn{4}{|c|}{ Saída/obstrução sonda } \\
\hline Sim & 1,72 & $1,21-2,45$ & 0,002 \\
\hline Não & 1,00 & & \\
\hline
\end{tabular}

Nota: UTI: Unidade de Terapia Intensiva. 
associou-se ao VRG, aos vômitos e aos sintomas gastrointestinais, enquanto a inadequação proteica associou-se somente à saída e obstrução de sonda, segundo variáveis de interrupção da dieta e complicações gastrointestinais (Tabela 2).

A Tabela 3 apresenta o modelo final da análise multivariada em ambos os desfechos. Verificou-se associação com inadequação calórica na categoria tempo de permanência em UTI $\leq 14$ dias ( $R P=2,07$ IC95\%:1,11-3,87) e presença de VRG (RP=2,41 IC95\%:1,51-3,86). Já a inadequação proteica esteve associada ao tempo de permanência em UTI $\leq 14$ dias ( $R P=2,05$ IC $95 \%: 1,14-3,67)$, à presença de VRG $(R P=1,54$ IC95\%:1,05-2,26) e à saída e obstrução da sonda $(\mathrm{RP}=1,72$ IC95\%:1,21-2,45). As demais variáveis selecionadas para a análise multivariada não mostraram associação após o ajuste final.

\section{I S C U S S Ã O}

Observou-se, neste estudo, uma alta prevalência de inadequação calórica e proteica, resultados semelhantes aos encontrados na literatura ${ }^{23-28}$. A luta contra o deficit de calorias e proteínas em pacientes de UTI com TNE exclusiva é diária. Alguns autores observaram que após a adoção de protocolo de nutrição enteral, a atuação da Equipe Multidisciplinar de Terapia Nutricional e atividades de educação continuada da equipe da UTI houve um aumento da razão dieta administrada/dieta prescrita ${ }^{26,28}$.

Em contrapartida, a presente pesquisa detectou um percentual médio de calorias e proteínas inferior ao recomendado na literatura. A utilização de fórmulas enterais com reduzido teor calórico e proteico (1,2 kcal/ml e $50 \mathrm{~g}$ de proteína/ $1000 \mathrm{~mL}$ ) pode ter colaborado com esses resultados, uma vez que aquelas com teores elevados favorecem o alcance da meta nutricional em menor tempo. O tempo ideal para atingir essa meta ainda precisa ser melhor estabelecido, pois depende das condições clínicas de cada paciente, bem como da logística de compra e abastecimento do local.
Apesar das particularidades de cada protocolo de estudo, uma diretriz recente ${ }^{29}$, juntamente com o consenso espanhol de Terapia Nutricional em cuidados intensivos ${ }^{30}$, sugerem que a meta de adequação de caloria e proteínas $>80 \%$ do valor estimado ou calculado deve ser alcançada em até 48-72 horas para que se possa atingir o benefício clínico durante a primeira semana de hospitalização. O algoritmo proposto pelo The PEP uP Protocol, em pacientes críticos, considera que caso esses valores não forem alcançados em até 72 horas e exista alto risco associado ao paciente, é indicado utilizar pro-cinéticos, bem como manter a sonda em posição intestinal, de forma a favorecer o alcance da meta nutricional ${ }^{31}$.

No presente estudo, as prevalências de inadequação calórica e proteica associaram-se ao tempo de internação. Mais da metade dos pacientes internados permaneceram um tempo menor na UTI ( $\leq 14$ dias) e, consequentemente, utilizaram a dieta enteral por menos tempo. Um estudo que avaliou a diferença entre a nutrição enteral prescrita e administrada a 85 adultos internados por 30 dias em um centro de terapia intensiva, verificou que o grupo o qual recebeu $>80 \%$ do volume de NE prescrita teve, em média, um maior tempo de uso da terapia nutricional enteral22. Campanella et al. ${ }^{24}$ observaram que quanto maior o número de dias em terapia enteral, maior a quantidade calórica e proteica consumida por dia. No presente estudo, a associação entre inadequação calórica e proteica e o menor tempo de UTI possivelmente se deu pelo maior tempo necessário para o alcance das necessidades nutricionais, devido à característica das fórmulas de nutrição enteral disponíveis na unidade no período.

O estudo de Oliveira et al. ${ }^{17}$ analisou os grupos segundo categorias de adequação percentual da oferta energética $(<70 \%, 70-90 \%$, $>90 \%$ ). Os autores verificaram que não houve diferença estatisticamente significativa quanto ao tempo de permanência na UTI. O'Meara et al. ${ }^{23}$, ao avaliarem os fatores associados às interrupções em nutrição enteral em pacientes críticos, obser- 
varam que esta foi interrompida em $27,3 \%$ do tempo disponível, sendo que a administração foi descontinuada em uma média de $6 \pm 0,9$ horas por paciente por dia. Na presente pesquisa, é possível que a administração descontinuada tenha ocorrido em tempo superior ao preconizado em protocolo da unidade (4 horas).

Observou-se, em metade da amostra, a presença de algum sintoma gastrointestinal. Além disso, a prevalência de inadequação calórica e proteica esteve associada ao volume residual gástrico elevado. Algumas intercorrências podem levar à administração inadequada da TNE nesses pacientes, sendo as complicações gastrointestinais as mais prevalentes devido à instabilidade hemodinâmica entre outros fatores inerentes às doenças de base ${ }^{14}$. Muitos estudos encontraram alta prevalência de complicações gastrointestinais $^{7-10,29,32,33}$, sendo o retorno gástrico elevado a complicação mais prevalente. Esses dados corroboram os achados deste estudo, apesar de nenhum dos autores citados ter avaliado a associação das complicações gastrointestinais com a inadequação calórica e proteica.

Na presente pesquisa, a prevalência de inadequação proteica foi maior em pacientes os quais tiveram a saída inadvertida/obstrução da sonda. Nas UTI estudadas, muitas vezes a repassagem da sonda não era realizada imediatamente e, além disso, a localização da mesma deveria ser confirmada através de um exame de Raios $X$ antes de se retomar a nutrição enteral. Outra situação a ser considerada é que essas interrupções podem não ser compensadas no mesmo dia. A adequação de proteína é, muitas vezes, realizada com a adição de módulos de proteínas à dieta ou à água, em frasco separado. No entanto, quando a administração de água para lavagem da sonda não é realizada corretamente, pode levar à obstrução da mesma. Além disso, a compensação da adequação da proteína demanda maior tempo quando comparada à adequação de calorias.

Na literatura, há uma grande divergência quanto aos valores de adequação considerados satisfatórios ou necessários para alcançar resultados clínicos positivos. Cahill et al. ${ }^{34}$, em estudo multicêntrico de 158 UTI de 30 países, consideraram arbitrariamente valores acima de $80 \%$ entre ofertado e prescrito como satisfatório, com nível de adequação alcançada de 45\%. Em contrapartida, Oliveira et al. ${ }^{17}$, analisando a adequação da oferta energética administrada em relação à quantidade prescrita em pacientes de UTI, consideraram como adequados valores acima de $70 \%$, sem que isso influenciasse a taxa de mortalidade. Krishnan et al. ${ }^{35}$ evidenciaram maior probabilidade de alta para os pacientes com adequação de 33 a $65 \%$ de oferta em relação às recomendações do guideline do American College of Chest Physicians.

A inadequação calórica e proteica observada nesses pacientes críticos em TNE pode contribuir significativamente para a incidência de desnutrição nessa população, bem como para um pior desfecho clínico. Outro ponto a ser discutido é o tempo de administração da dieta enteral. No presente estudo, não foi avaliada a média de horas que o paciente ficou sem recebê-la. No protocolo das unidades do estudo, a velocidade de infusão é programada considerando quatro horas de interrupção da dieta enteral. Vale destacar que, dentre os pacientes estudados, $86,84 \%$ apresentaram intercorrências no período avaliado, sendo o jejum presente em mais da metade dos daqueles com TNE. A velocidade de infusão programada pode não ter sido compatível com a necessidade de interrupção da oferta da dieta enteral, porém muitos profissionais optam por prescrever uma velocidade de infusão mais lenta e evoluir cuidadosamente a dieta. Dessa maneira, a aplicação desses indicadores na TNE é uma forma de avaliação que permite a monitorização da qualidade da assistência prestada e evidência uma necessidade de conscientização dos profissionais de saúde a respeito da importância de se estabelecer medidas as quais visem a redução de complicações gastrointestinais, de interrupção da dieta, bem como a revisão do protocolo de administração da dieta enteral. 


\section{O N CLUSÃ O}

Os resultados deste estudo indicam que o percentual médio de calorias e proteínas administradas por meio da terapia nutricional enteral aos pacientes críticos não alcançou a meta estabelecida. A prevalência de inadequação calórica e proteica na amostra foi alta e esteve associada às duas primeiras semanas de internação, bem como a complicações gastrointestinais. A saída ou obstrução da sonda parece causar prejuízo na administração proteica.

Essa inadequação, associada a fatores de interrupção da nutrição enteral, pode contribuir signicativamente para incidência de desnutrição energético-proteica em paciente de UTI e para o aumento da prevalência de morbidade e mortalidade. Portanto, o conhecimento dos fatores que impedem a efetiva administração energético-proteica permite a adoção de medidas as quais visem intervir com mais eficácia na conduta nutricional e melhorar o quadro clínico do paciente crítico. Com isso, o suporte nutricional pode ajudar a diminuir consideravelmente a prevalência de desnutrição em UTI e, assim, contribuir decisivamente para a recuperação do estado clínico do paciente. Dessa maneira, os achados deste estudo podem colaborar com a elaboração e implantação de protocolos de UTI por equipe multiprofissional que vise medidas para otimizar a administração satisfatória de caloria e proteínas durante o período de internação.

\section{COLABORADORES}

Todos os autores contribuíram na concepção e desenho do estudo, análise de dados e redação final.

\section{RE FER Ê N CIAS}

1. Jensen GL, Wheeler D. A new approach to defining and diagnosing malnutrition in adult critical illness. Curr Opin Crit Care. 2012; 18(2):206-11. http:// dx.doi.org/10.1097/MCC.0b013e328351683a

2. Projeto Diretrizes. Terapia Nutricional no Paciente Grave. São Paulo: Sociedade Brasileira de Nutrição Enteral e Parenteral; 2011 [acesso 2016 mar 10]. Disponível em: http://www.projetodiretrizes.org.br/ 9_volume/terapia_nutricional_no_paciente_ grave.pdf
3. Ribeiro LMK, Oliveira Filho RS, Caruso L, Lima PA, Damasceno NRT, Soriano FG. Adequação dos balanços energético e proteico na nutrição por via enteral em terapia intensiva: quais são os fatores limitantes? Rev Bras Ter Intensiva. 2014; 26(2):155-62. http://dx.doi.org/10.5935/0103-507X.20140023

4. Hegazi RA, Wischmeyer PE. Clinical review: Optimizing enteral nutrition for critically ill patients - a simple data-driven formula. Crit Care. 2011; 15(6):1385-8. http://dx.doi.org/10.1186/cc10430

5. Kreymann KG, Berger MM, Deutzc NEP, Hiesmayrd $M$, Jolliete $P$, Kazandjiev $G$, et al. ESPEN guidelines on enteral nutrition: Intensive care. Clin Nutr. 2006; 25(2):210-23. http://dx.doi.org/10.1016/j.clnu. 2006.01.021

6. Doig GS, Simpson F, Sweetman EA, Finfer SR, Cooper DJ, Heighes PT, et al. Early parenteral nutrition in critically ill patients with short-term relative contraindications to early enteral nutrition: A randomized controlled trial. JAMA. 2013; 309(20):2130-8. http://dx.doi.org/10.1001/jama. 2013.5124

7. American Society for Parenteral and Enteral Nutrition. Guidelines for the provision and assessment of nutritional support therapy in the adult critically ill patients. J Parenter Enteral Nutr. 2009; 33(3):277-316. http://dx.doi.org/10.1177/ 0148607109335234

8. Oliveira SM, Burgos MGPA, Santos EMC, Prado LVS, Petribu MMV, Bonfim FMTS. Complicações gastrintestinais e adequação calórico-proteica de pacientes em uso de nutrição enteral em uma unidade de terapia intensiva. Rev Bras Ter Intensiva. 2010; 22(3):270-3. http://dx.doi.org/10.1590/S0 103-507X2010000300009

9. Agudelo GM, Giraldo NA, Román J, Serna A, Hoyos $\mathrm{M}$, Aguilar $\mathrm{N}$, et al. Incidencia de complicaciones del soporte nutricional en pacientes críticos: Estudio multicêntrico. Nutr Hosp. 2011; 26(5):537-45.

10. Sant'ana IES, Mendonça SS, Marshall, NG. Adequação energético-proteica e fatores determinantes na oferta adequada de nutrição enteral em pacientes críticos. Com Ciên Saúde. 2013; 22(4):47-56.

11. Heidegger $C P$, Darmon P, Pichard C. Enteral VS. Parenteral nutrition for the critically ill patient: $A$ combined support should be preferred. Curr Opin Crit Care. 2008; 14(4):408-14. http://dx.doi.org/ 10.1097/MCC.0b013e3283052cdd

12. Oliveira-Filho RS, Tamburrino AC, Trevisani VS, Rosa VM. Main barriers in control of energy-protein deficit in critical oncologic patient at nutritional risk. J Integr Oncol. 2016; 5(1):156. http://dx.doi.org/ 10.4172/2329-6771.1000156

13. Villet $S$, Chiolero RL, Bollmann MD, Revelly JP, Cayeux RNMC, Delarue J, et al. Negative impact of hypocaloric feeding and energy balance on clinical 
outcome in ICU patients. Clin Nutr. 2005; 25(4):502-9. http://dx.doi.org/10.1016/j.clnu. 2005.03.006

14. Seron-Arbeloa C, Zamora-Elson M, LabartaMonzon L, Mallor-Bonet T. Enteral nutrition in critical care. J Clin Med Res. 2013; 5(1):1-11. http://dx. doi.org/10.4021/jocmr1210w

15. Thibault R, Pichard C. Nutrition and clinical outcome in intensive care patients. Curr Opin Clin Nutr Metab Care. 2010; 12(2):177-83. http://dx.doi.org/ 10.1097/MCO.0b013e32833574b9

16. Aranjues AL, Teixeira ACC, Caruso L, Soriano FG. Monitoração da terapia nutricional enteral em UTI: indicador de qualidade? Mundo da Saúde. 2008; 32(1):16-23.

17. Oliveira NS, Caruso L, Bergamaschi DP, Cartolano FC, Soriano FG. Impacto da adequação da oferta energética sobre a mortalidade em pacientes de UTI recebendo nutrição enteral. Rev Bras Ter Intensiva. 2011; 23(2):183-9. http://dx.doi.org/10.1590/S0 $103-507 \times 2011000200011$

18. Boullata J, Carney LN, Guenter P. Enteral nutrition handbook. 2a ed. Silver Spring: Aspen; 2010.

19. Detsky AS, McLaughlin JR, Baker JP, Johnston N, Whittaker S, Mendelson RA, et al. What is subjective global assessment of nutritional status? J Parenter Enteral Nutr. 1987; 11(1):8-13.

20. Chumlea WC, Guo SS, Roche AF, Steinbaugh ML. Prediction of body weight for the nonambulatory elderly from anthropometry. J Am Diet Assoc. 1988; 88(5):563-8.

21. Couto CFL, Moreira JS, Hoher JA. Terapia nutricional enteral em politraumatizados sob ventilação mecânica e oferta energética. Rev Nutr. 2012; 25(6):695-705. http://dx.doi.org/10.1590/\$1415-5 2732012000600002

22. Assis MCS, Silva SMR, Leaes DM, Novello CL, Silveira CRM, Mello ED, et al. Nutrição enteral: diferenças entre volume, calorias e proteínas prescritos e administrados em adultos. Rev Bras Ter Intensiva. 2010; 22(4):346-50. http://dx.doi.org/10.1590/S01035 07X2010000400006

23. O'Meara D, Mireles-Cabodevila E, Frame F, Hummell AC, Hammel J, Dweik RA, et al. Evaluation of delivery of enteral nutrition in critically ill patients receiving mechanical ventilation. Am J Crit Care. 2008; 17(1):53-61.

24. Campanella LCA, Silveira BM, Rosário Neto O, Silva AA. Terapia nutricional enteral: a dieta prescrita é realmente infundida? Rev Bras Nutr Clin. 2008; 23(1):21-5

25. Teixeira ACC, Caruso L, Soriano F. Terapia nutricional enteral em unidade de terapia intensiva: infusão versus necessidades. Rev Bras Ter Intensiva. 2006; 18(4):331-7.
26. Campos DJ, Silva AFF, Souza MH, Shieferdecker ME. Otimização do fornecimento calórico-proteico na terapia de nutrição enteral em unidade de terapia intensiva com o uso de protocolo. Rev Bras Nutr Clin. 2006; 21(1):2-5

27. Isidro MF, Lima DSC. Adequação calórico-proteica da terapia nutricional enteral em pacientes cirúrgicos. Rev Assoc Med Bras. 2012; 58(5):580-6. http://dx.doi.org/10.1590/S0104-4230201200 0500016

28. Oliveira NS, Caruso L, Soriano FG. Terapia nutricional enteral em UTI: seguimento longitudinal. Nutrire: Rev Soc Bras Alim Nutr. 2010; 35(3):133-48.

29. McClave AS, Martindale RG, Vanek VW, McCarthy M, Roberts P, Taylor B, et al. Guidelines for the provision and assessment of nutrition support in the adult critically ill patient: Society of critical care medicine (SCCM) and american society for parenteral and enteral nutrition (ASPEN). J Parenter Enteral Nutr. 2016;40(2):159-211. http://dx. doi.org/10.1177/0148607109335234

30. Fernández Ortega JF, Meseguer JH, García PM. Guidelines for specialized nutritional and metabolic support in the critically-ill patient. Update. Consensus SEMICYUC-SENPE: Indications, timing and routes of nutrient delivery. Nutr Hosp. 2011; 26(Suppl. 2):7-11. http://dx.doi.org/10.1590/S0 212-16112011000800009

31. Heyland DK, Cahill NE, Dhaliwal R, Wang M, Day $A G$, Alenzi $A$, et al. Enhanced protein-energy provision via the enteral route in critically ill patients: A single center feasibility trial of the PEP uP protocol. Crit Care. 2010; 14(2):R78. http://dx.doi.org/10. $1186 /$ cc8991

32. Borges RM, Nonino-Borges CB, Campos AD, Basile-Filho A. Incidência de complicações em terapia nutricional enteral de pacientes em estado grave. Rev Bras Ter Intensiva. 2005;17(2):98-103.

33. Agudelo GM, Giraldo NA, Román J, Serna A, Hoyos M, Aguilar N, et al. Incidencia de complicaciones del soporte nutricional en pacientes críticos: estudio multicêntrico. Nutr Hosp. 2011; 26(5):537-45. http:// dx.doi.org/10.3305/nh.2011.26.3.4789

34. Cahill NE, Dhaliwal R, Day AG, Jiang X, Heyland D. Nutrition therapy in the critical care setting: What is "best achievable" practice? An international multicenter observational study. Crit Care Med. 2010; 38(2):395-401. http://dx.doi.org /10.1097/ CCM.0b013e3181c0263d

35. Krishnan JA, Parce PB, Martinez A, Diette GB, Brower RG. Caloric intake in medical ICU Patients. Chest. 2003;124(1):297-305.

Recebido: agosto 20, 2015 Versão final: junho 9, 2016 Aprovado: junho 24, 2016 